\title{
The Three-Dimensional Structure of (+39) RNA-Polymerase Elongation Complex Determined by Cryo-Electron Microscopy
}

\author{
Oleg V. Chertkov ${ }^{1}$, Maria G. Karlova ${ }^{1}$, Vasily M. Studitsky ${ }^{1,2}$ and Olga S. Sokolova ${ }^{1 *}$ \\ 1. Biology Faculty, Lomonosov, Moscow State University, Moscow, Russia. \\ 2. Fox Chase Cancer Center, Philadelphia, PA, USA. \\ * Corresponding author: sokolova@mail.bio.msu.ru
}

The nucleosome is a histone octamer (central heterotetramer $(\mathrm{H} 3-\mathrm{H} 4)_{2}$ and two $\mathrm{H} 2 \mathrm{~A}-\mathrm{H} 2 \mathrm{~B}$ heterodimers) linking a double-stranded DNA of $147 \mathrm{bp}$. The structure of the nucleosome was determined by X-ray analysis [1]. The structure of DNA-dependent RNA polymerases is identical for prokaryotes and eukaryotes. The holoenzyme of Escherichia coli RNA polymerase consists of a "core" enzyme including 5 subunits $\left(\beta-, \beta^{\prime}-, 2 \alpha-, \omega-\right)$ which has catalytic activity and a sigma factor that recognizes the starting point of transcription [2].

During the passage of the RNA polymerase through the region of the nucleosomal DNA localized 45-50 from the promoter-proximal nucleosome boundary, the RNA polymerase can induce the formation of a small-sized intra-nucleosomal loop. This complex was called the "zero-size" loop (or Ø-loop), since DNA-histone surround the transcribing RNA polymerase (Fig. 1). The formation of a Ø-loop can induce a partial detachment of the DNA from the surface of the octamer and the loss of the dimer H2A-H2B. Øloop formation is necessary for transcription without the dissociation of $\mathrm{H} 3-\mathrm{H} 4$ core histones from the DNA and, possibly, plays a role in maintaining the histone code in the process of transcription. It has been shown by the footprinting that the $\varnothing$-loop can be formed only when the RNA polymerase is in position +39 or +49 in the nucleosome [3-4].

In our study, the structure of the elongation complex of RNA-polymerase with the nucleosome in position +39 was analyzed by cryo- and negative stain EM. All protein and DNA-protein complexes $(E$. coli RNAP and -H1 donor chromatin) were purified as described [5]. The DNA template consists of two parts: the nucleosome positioning sequence s603 and the T7A1 promoter. The DNA sequence was modified to form a region that does not contain a base $\mathrm{T}$ from the start of the transcription to position +39. This sequence of nucleotides allowed to obtain an elongation complex with an RNA-polymerase stalled at position of +39 base pairs from entering the nucleosomal DNA during transcription, in the presence of a limited set of ribonucleotides. The nucleosome reconstitution, the assembly of the elongation complex of the RNA-polymerase with the nucleosome and its purification on the affinity monolayer were carried out as described elsewhere [6-7].

We obtained the negative stained images (Fig. 2F) using a JEOL2100 (JEOL) and the cryo-EM images using a Technai F20 (FEI) cryo-microscope (Fig. 2B). With the help of the random conical tilt method, about 10,000 individual particles were collected, subjected to reference-free classification and single particle analysis. The 3D reconstruction was calculated using RELION2.1, which combined two interconnected domains (Fig. 2C). The sizes of these domains are comparable with the crystal structures of RNA polymerase and nucleosome (Fig. 2D), which indicates the absence of fundamental disturbances in the structure of the complex [8]. 
References:

[1] M Dion et al., Science 315 (2007), p. 1405.

[2] KS Murakami and SA Darst, Curr. Opin. Struct. Biol. 13 (2003), p. 31.

[3] OI Kulaeva et al., Nat. Struct. Mol. Biol. 16 (2009), p. 1272.

[4] HW Chang et al., Trends Cell. Mol. Biol. 8 (2013), p. 13.

[5] DA Gaykalova et al., Methods Enzymol. 512 (2012), p. 293.

[6] OV Chertkov et al., Moscow Univ. Biol. Sci. Bull. 73 (2018), p. 142.

[7] MG Karlova et al., Russ. J. Bioorg. Chem. 43 (2017), p. 637.

[8] The authors acknowledge funding from the RSF (19-74-30003). The Technai F20 microscope is part of Brandeis University EM facility and JEOL2100 microscope is part of User facility center of MSU "Electron microscopy in life sciences".

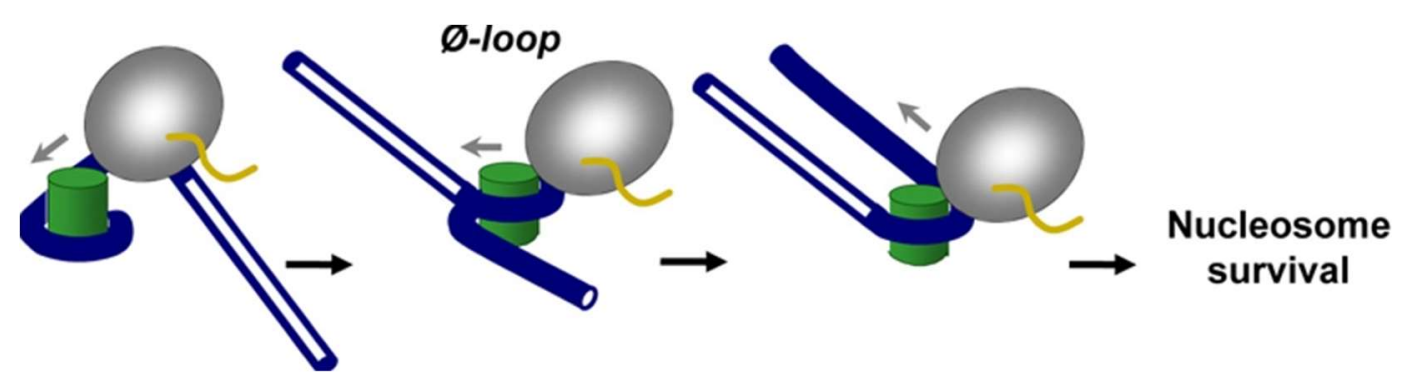

Figure 1. The formation of a stable intranucleosomal $\varnothing$-loop-containing intermediate at position +39 (from [4]).
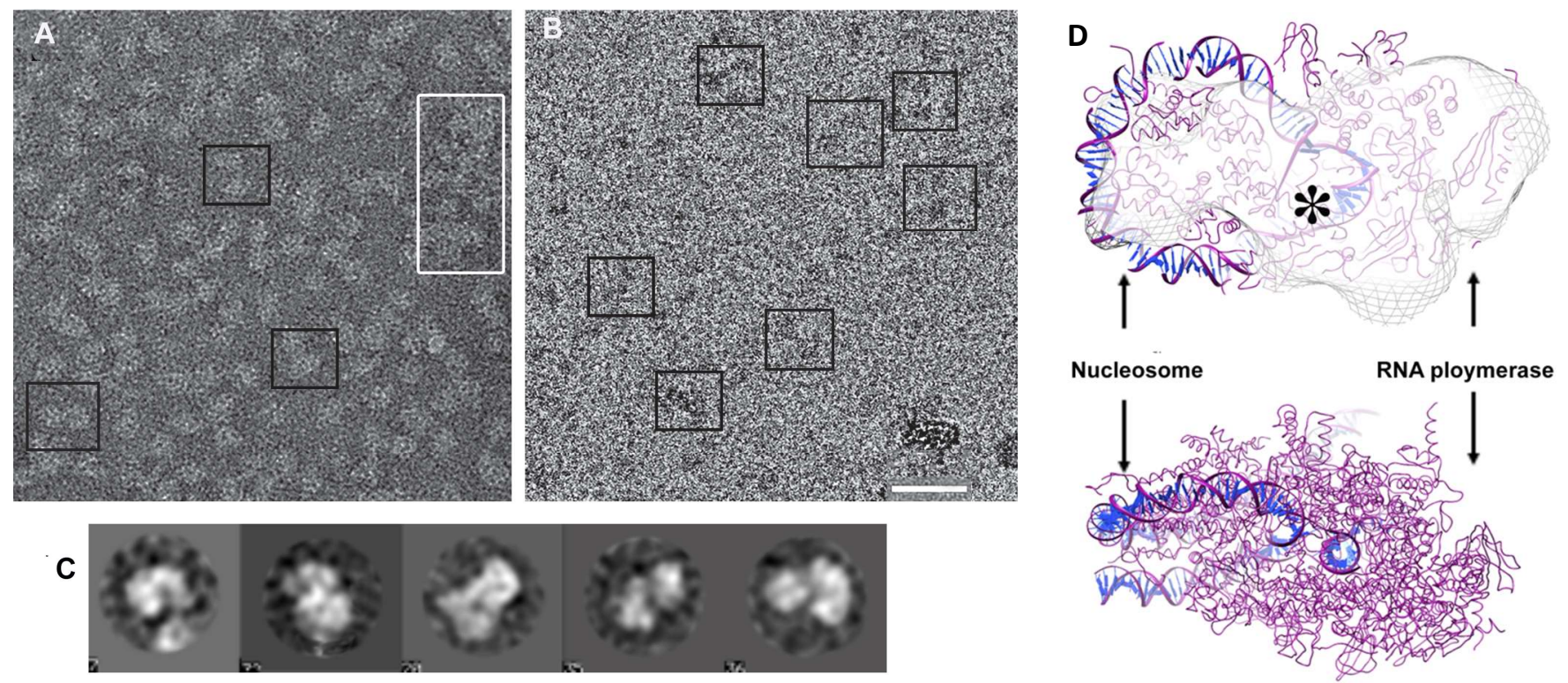

Figure 2. Electron microscopical study of an elongation complex of RNA-polymerase with the nucleosome in position +39 . (A) - negative stain; (B) - cryo-EM. (C) 2D class-averages of +39 complexes. (D) The crystal structures of the RNA polymerase and nucleosome, docked into the overall $3 \mathrm{D}$ density of the +39 complex, indicate the position of the Ø-loop (star). Bar $-100 \mathrm{~nm}$. 